\title{
Highly Sensitive
}

\section{Electrochemiluminescent Nanobiosensor for the Detection of Palytoxin}

\author{
Valeria Anna Zamolo, ${ }^{+, \uparrow}$ Giovanni Valenti, ${ }^{ \pm, q}$ Enrica Venturelli, ${ }^{\S}$ Olivier Chaloin, ${ }^{\S}$ Massimo Marcaccio, ${ }^{\ddagger}$ \\ Sabrina Boscolo, ${ }^{\perp}$ Valentina Castagnola, ${ }^{\ddagger}$ Silvio Sosa, ${ }^{\prime \prime}$ Federico Berti, ${ }^{\dagger}$ Giampaolo Fontanive, ${ }^{\dagger}$ Mark Poli, ${ }^{\Delta}$ \\ Aurelia Tubaro,",* Alberto Bianco, ${ }^{\text {s,* }}$ Francesco Paolucci, ${ }^{\ddagger, *}$ and Maurizio Prato ${ }^{\dagger, *}$ \\ ${ }^{\dagger}$ Center of Excellence on Nanostructured Materials, Department of Chemical and Pharmaceutical Sciences, and INSTM, Unit of Trieste, University of Trieste, \\ P.le Europa 1, 34127 Trieste, Italy, ${ }^{\ddagger}$ Department of Chemistry "G. Ciamician", University of Bologna, Via F. Selmi 2, 40126 Bologna, Italy, , Laboratoire d'Immunologie \\ et Chimie Thérapeutiques, CNRS, Institut de Biologie Moléculaire et Cellulaire, 67000 Strasbourg, France, ${ }^{\perp}$ Department of Life Sciences, University of Trieste, \\ Via L. Giorgieri 5, 34127 Trieste, Italy, "Department of Life Sciences, University of Trieste, Via A. Valerio 6, 34127 Trieste, Italy, and ${ }^{\triangle}$ U.S. Army Medical Research \\ Institute of Infectious Diseases, Ft Detrick, Maryland 21701-5011, United States. "These authors contributed equally to this work.
}

ood safety matters constantly attract
regulatory authorities and adverse
public attention due to their potentially widespread impact on human health. The design and development of increasingly sensitive devices able to detect contaminants in foods and/or in the food chain is therefore an issue of vital importance. In this context, the increasing diffusion of palytoxin (PITX) ${ }^{1}$ led us to investigate the possibility of developing an innovative electrochemiluminescence-based biosensor for the detection of this potent marine toxin. PITX and its analogues are known worldwide for their extreme toxicity and ability to contaminate seafood, causing intoxications and/or fatalities after ingestion. ${ }^{2}$

Despite the increasing reports of PITXcontaminated seafood in temperate waters (i.e., Mediterranean Sea), there are no validated and accepted protocols for the detection and quantification of this class of biomolecules, while the existing procedures present limitations in term of sensitivity and specificity. ${ }^{3}$ These challenges led us to design a novel hybrid system for PITX detection, in which sandwich immunoassay and electrochemiluminescence $(E C L)^{4,5}$ were successfully combined. The unique ability of an antibody to specifically recognize its antigen together with the ECL-based detection approach presents several advantages, such as limited costs, inexpensive equipment, rapidity of analysis, and easy use. ${ }^{6,7}$ The sensitivity of the immunosensor was enhanced by interfacing the transduction
ABSTRACT Marine toxins appear to be increasing in many areas of the world. An emerging problem in the Mediterranean Sea is represented by palytoxin (PITX), one of the most potent marine toxins, frequently detected in seafood. Due to the high potential for human toxicity of PITX, there is a strong

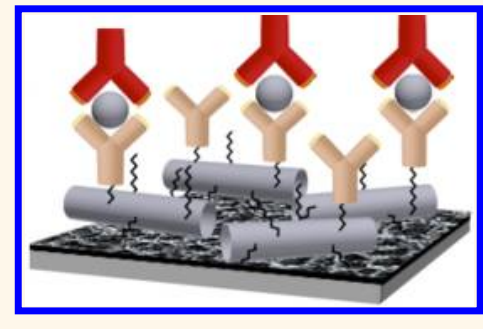
and urgent need for sensitive methods toward its detection and quantification. We have developed an ultrasensitive electrochemiluminescence-based sensor for the detection of PITX, taking advantage of the specificity provided by anti-PITX antibodies, the good conductive properties of carbon nanotubes, and the excellent sensitivity achieved by a luminescencebased transducer. The sensor was able to produce a concentration-dependent light signal, allowing PITX quantification in mussels, with a limit of quantification ( $L 0 Q=2.2 \mu \mathrm{g} / \mathrm{kg}$ of mussel meat) more than 2 orders of magnitude more sensitive than that of the commonly used detection techniques, such as LC-MS/MS.

KEYWORDS: biosensor · palytoxin · carbon nanotubes · antibody · electrochemiluminescence $\cdot$ shellfish

component, an optically transparent electrode (OTE), with functionalized multiwalled carbon nanotubes (f-MWCNTs) (Figure 1).

Indeed, carbon nanotubes (CNTs) have been already proposed as excellent candidates to improve (bio)sensor performances, as they display remarkable electronic properties $^{8-11}$ and the ability to act as individual nanoelectrodes. $^{12-14}$ For example, Heller et al. have proposed a multiplexed biosensor based on single-walled CNTs modified with appropriate nucleic acid sequences. This

DNA-based sensor allowed the identification @ 2012 American Chemical Society

\section{* Address correspondence to tubaro@units.it, a.bianco@ibmc-cnrs.unistra.fr, francesco.paolucci@unibo.it, prato@units.it.}

Received for review June 11, 2012

Published online August 22, 2012 10.1021/nn302573c and accepted August 22, 2012. 
of different classes of molecules (i.e., alkylating agents and reactive oxygen species). ${ }^{15}$ Moreover, CNTs present a high surface area and wide versatility in functionalization. ${ }^{12,16}$ These characteristics were exploited to prepare doubly functionalized CNTs. Using a combination of amidation and 1,3-dipolar cycloaddition reactions, we have introduced amino groups both onto the sidewalls and at the tips of oxidized CNTs. Through a selective chemical ligation strategy ${ }^{17}$ the amino groups at the CNT sidewalls were derivatized to covalently bind the anti-PITX monoclonal antibody $\left(m A b_{1}\right)$, whereas the groups at tips were exploited to anchor the immunoconjugate onto the surface of the electrode (Figure 1a). The electrochemiluminescent device was completed by preparing a ruthenium-based complex linked to an anti-PITX polyclonal antibody $\left(\mathrm{pAb}_{2}\right)$. The quantitative detection of PITX was achieved in a controlled and reproducible manner by measuring the variation in the intensity of the emitted light under excitation conditions. Finally, the utility of our approach was demonstrated by PITX detection in complex matrices, such as mussels and microalgae. The biosensor we report here presents a detection limit within sub-nanograms per milliliter of solution, that is, 1 to 2 orders of magnitude lower than the values reported for the most quoted analytical

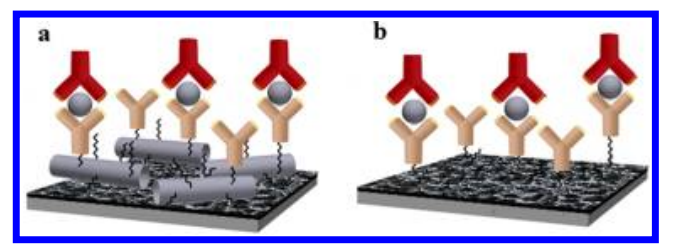

Figure 1. Schematic representation of the antibody-CNTbased immunosensor (a) and the control antibody-based immunosensor devoid of CNTs (b), developed in this work. Immunosensors are sketched with an optically transparent electrode covered by the polymer layer and the immunoassay assembled on it, in which PITX (gray sphere) is specifically recognized by the capture antibody (beige) linked to the nanotubes or directly grafted to the polymer and quantified by the detection antibody (red). techniques for PITX, i.e., liquid chromatography coupled to mass spectrometry or fluorescence polarization. ${ }^{3,18,19}$

\section{RESULTS AND DISCUSSION}

Preparation of the CNT-Based Immunosensing Component. Electrochemiluminescence, which means chemiluminescence produced by electrogenerated species in solution, is known to offer high sensitivity and sensibility in many applications. $^{6-8}$ The electrochemically induced emission opens the possibility of avoiding excitation sources and guarantees remarkably low noise, signal specificity, and easy design of the device. For all these reasons, ECL commercial assays are widely used in very important areas, such as clinical diagnosis, food and water testing, environmental monitoring, and biowarfare agent detection. ${ }^{7}$

Our ECL device comprises several elements. The immunosensing component is constituted by $f$-MWCNTs, modified with an anti-PITX monoclonal antibody. The strategy to prepare this new construct uses a double functionalization approach of the nanotubes. We combined the versatile 1,3-dipolar cycloaddition reaction with the amidation reaction mainly occurring at the oxidized CNT tips and defects (Scheme 1). The ammonium-derivatized pyrrolidine rings around the sidewalls of the nanotubes were covalently linked to the immunoglobulin, while the linker inserted at the open ends of the tubes allowed their anchoring onto the electrode surface. For this purpose, purified MWCNTs were first shortened by an oxidation reaction in acidic conditions. Subsequently, MWCNTs (1) were functionalized with ammonium groups through 1,3-dipolar cycloaddition of in situ generated azomethine ylides (2). The amino groups were allowed to react with a heterobifunctional cross-linker (3-maleimidopropionic $N$-hydroxysuccinimide ester) to introduce the maleimido moieties necessary to link the antibody (compound $\mathbf{3}$ ). The carboxylic groups were converted to the corresponding amides (4) through condensation with Boc-monoprotected diaminotriethylene glycol. Finally by using a chemically selective ligation approach, $m A b_{1}$ was coupled to maleimidofunctionalized MWCNTs (conjugate 5). ${ }^{17}$ This approach

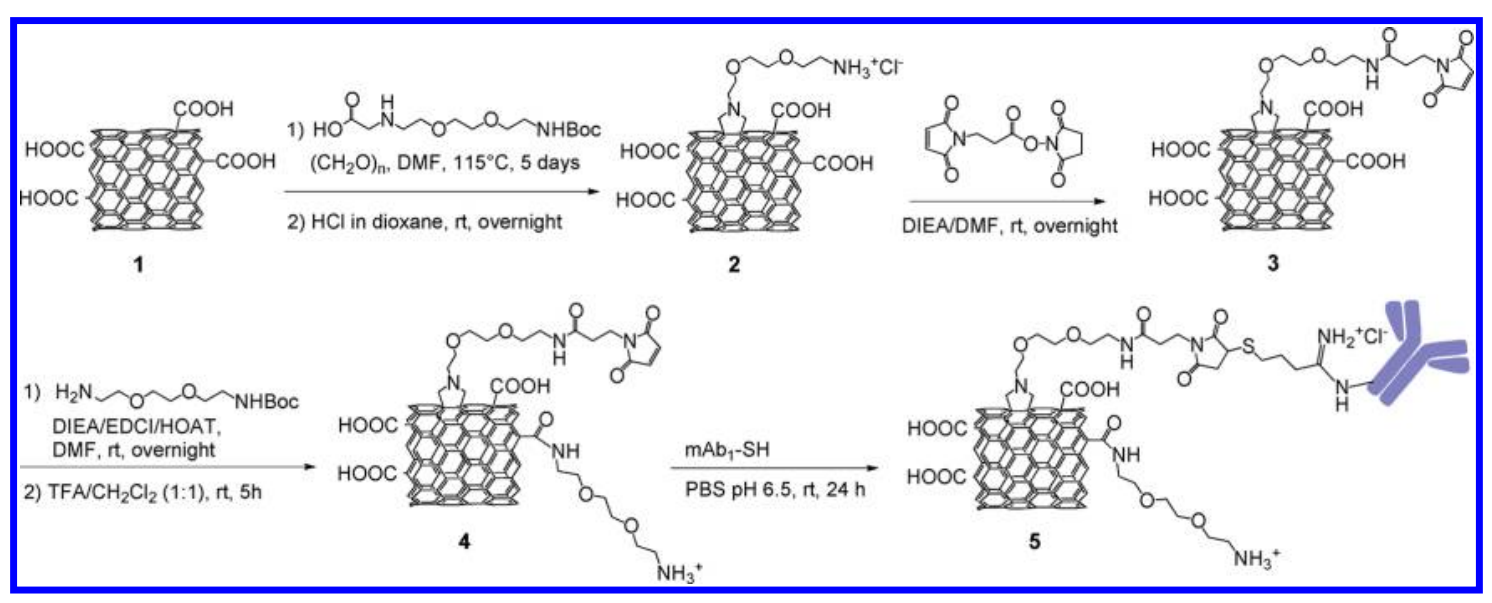

Scheme 1. Preparation of Doubly Functionalized MWCNT 5 


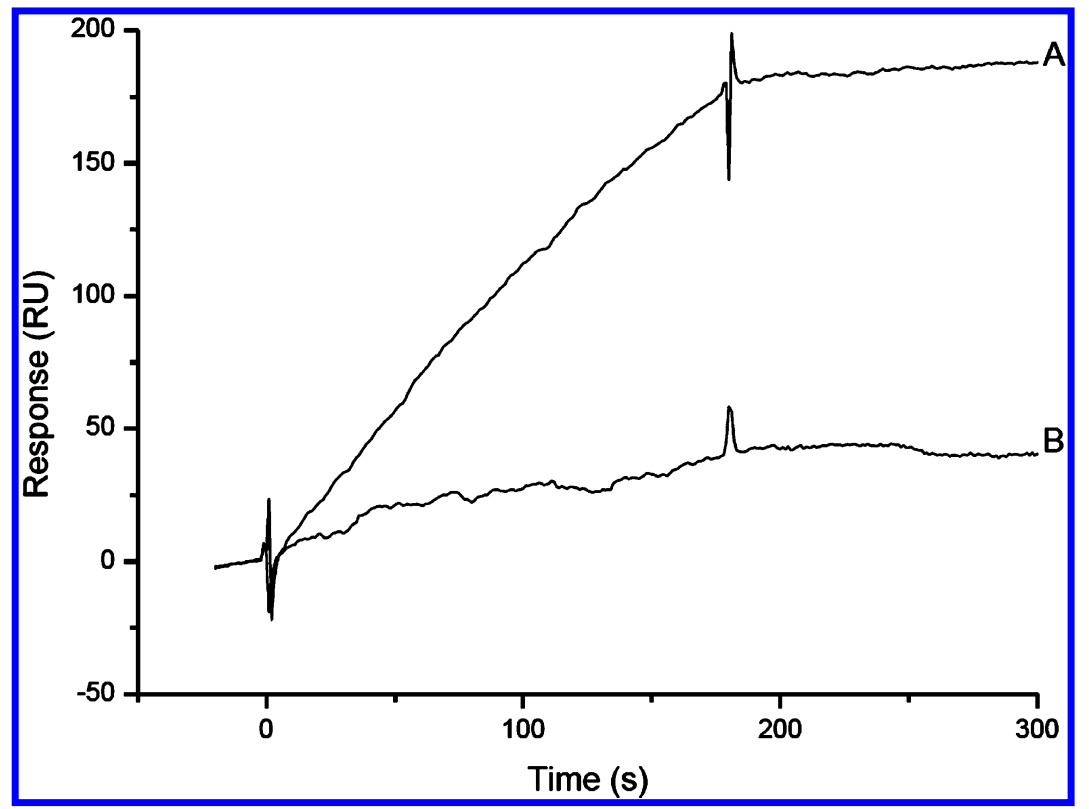

Figure 2. SPR analysis performed on biotin-PITX using MWCNT-mAb conjugate (5). Concentrations: $100 \mu \mathrm{g} \mathrm{mL}^{-1}(\mathrm{~A})$ and $50 \mu \mathrm{g} \mathrm{mL}^{-1}$ (B).

confers high chemical stability to the construct by limiting at the same time the presence of adsorbed antibodies that could be released from the nanotube surface, rendering the system unstable. Moreover, this strategy offers a good control over the entire process, as the two functional groups were attached at noncompeting positions.

The obtained $f$-MWCNTs (5) were characterized by several techniques, including transmission electron microscopy (TEM), thermogravimetric analysis (TGA), gel electrophoresis, and surface plasmon resonance (SPR) (see Supporting Information for details). In particular, SPR analysis allowed us to assess the affinity of the CNT-coupled antibody toward its antigen. ${ }^{20,21}$ For this purpose, we immobilized biotinylated-PITX (biotin-PITX) on the sensor chip through a streptavidin-biotin complex, while the monoclonal antibody or the MWCNT$m A b_{1}$ (5) flowed over the chip. The affinity was determined by measuring the equilibrium dissociation constant, $K_{\mathrm{D}}$. The value for the interaction between the $\mathrm{mA} \mathrm{b}_{1}$ alone and its antigen was $K_{\mathrm{D}}=2.46 \times 10^{-10} \mathrm{M} \cdot{ }^{21}$ We then evaluated the binding of the MWCNT- $m A_{1}$ (5) with biotin-PITX (Figure 2).

The CNT-immunoconjugate was again able to recognize the antigen on the sensor chip. In this case it was not possible to calculate quantitative binding from the SPR, for the following reasons: (i) a precise molecular weight for CNTs (being a heterogeneous material) cannot be determined; therefore the $K_{\mathrm{D}}$ value could not be obtained from the sensorgrams; and (ii) the antibody conjugation is likely to be unevenly distributed along the nanotubes, which may affect its antigenbinding capacity. However, the SPR curves allowed us to prove that the covalent attachment of the antibody onto CNTs did not affect its recognition capacity, demonstrating that the conjugate can be used to specifically recognize palytoxin once inserted in the biosensor (vide infra).

Preparation of the Transduction and Detecting Components. The next step in the fabrication of the biosensing platform was the realization of the transduction component consisting of an optically transparent electrode (OTE) characterized by a surface modified with an electrochemically grown layer of $\mathrm{N}$-succinimidyl polyacrylate (PNSA) (Figure 3). The chemisorption of polymers onto conducting surfaces, originally reported for acrylonitrile, was then extended to various substituted methacrylates with the additional advantage of tuning the surface functionality and reactivity by the proper choice of the ester group of the monomer. Here, we have chosen $\mathrm{N}$-succinimidyl acrylate (NSA), which bears activated ester groups and is highly reactive toward nucleophiles and hence rather versatile for postfunctionalization, even for dispersions of CNT, of the grafted polymeric chains (see Experimental Section and Supporting Information). ${ }^{22,23}$

In a typical experiment, the polymerization was induced by cyclic voltammetry scanning the potential until the first reduction of the monomer (usually for indium tin oxide (ITO) substrates: $-2.1 \mathrm{~V}$ ). The formation of the chemisorbed insulating film was accompanied by a decrease in the cathodic current, and the best performance of deposited films was obtained when stopping at $\sim 40 \%$ of the initial current.

The last step in the preparation of the biosensor was the synthesis of the detecting component $\left(\mathrm{pAb}_{2}-\mathrm{Ru}\right)$, responsible for the detection of the analyte. This moiety consisted of polyclonal anti-PITX antibodies $\left(\mathrm{pAb}_{2}\right)$ coupled to the ruthenium tris-bipyridine complex, the ECL label. Polyclonal antibodies, generated against PITX, 


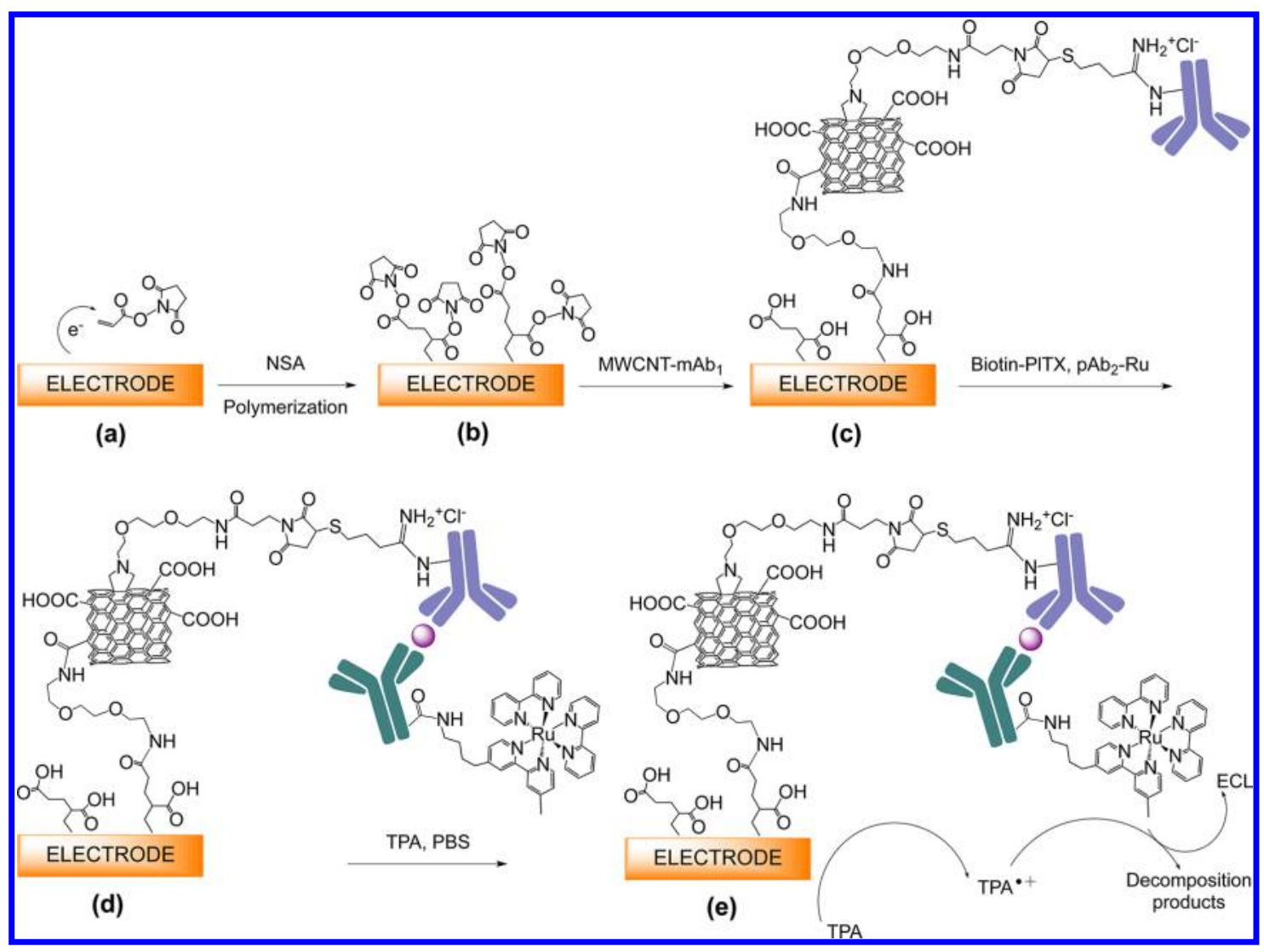

Figure 3. (a) Electrografting of ITO with NSA. (b) Functionalization of MWCNT-mAb . (c) Addition of biotin-PITX (purple sphere) followed by $\mathrm{PAb}_{2}$-Ru. (d) Addition of the TPA co-reactant. (e) ECL development.

are able to recognize different epitopes of the toxin and thus simultaneously bind to the toxin complexed to $m A b_{1}$, whereas the fluorophore is responsible for the production of the ECL signal. The ECL label was covalently linked to $\mathrm{pAb}_{2}$ via amide bond formation between the carboxylic groups naturally present on the immunoglobulins and the free terminal amino groups of the fluorophore (see Supporting Information). Ruthenium polypyridine complexes are, thanks to their good combination between electrochemical stability and photophysical proprieties, well-known and robust ECL fluorophores. For these reasons $\mathrm{Ru}(\mathrm{bpy})_{3}{ }^{2+}$ and its derivatives are the most common employed luminophores for commercial applications.

Biosensor Assembly and Response to Biotin-PITX. Once the different components of the biosensor were developed and characterized (see Supporting Information), we proceeded with the assembly of the immunosensor by anchoring onto the electrografted polymer the nanotubes covalently functionalized with the monoclonal antibody (MWCNT-mAb 15 ) (Figure 3). The conjugate, dispersed in phosphate-buffered saline (PBS), was deposited onto the electrode at a concentration of $1 \mathrm{mg} \cdot \mathrm{mL}^{-1}$ and incubated overnight at $4{ }^{\circ} \mathrm{C}$. Subsequently, all nonspecific binding sites were saturated by BSA (bovine serum albumin) and washed with the detergent Tween-20 (see Experimental Section and Supporting Information). This procedure represents an important control to test the stability of the immunosensing interface since analogous electrodes, prepared by simple drop casting of antibody-modified carbon nanotubes onto ITO surfaces, did not survive the treatment with the detergent. ${ }^{24}$ The medium was then removed and replaced with a solution of biotin-PITX at a concentration of $260 \mathrm{ng} \cdot \mathrm{mL}^{-1}$ for one hour. We note that, due to the conformational features of PITX in aqueous solutions, which is present as an associated dimer, ${ }^{25}$ the device was developed using PITX conjugated to biotin via the primary amino groups (see Experimental Section for the details) to prevent dimerization. In principle, this causes no problems, since PITX and biotin-PITX possess the same binding affinity for the $m A b_{1}$. However, in view of its practical use, the working range of the biosensor and its suitability for PITX detection in mussels and microalgae was evaluated using the commercially available toxin.

Finally, the system was exposed to the polyclonal antibody labeled with the electrochemiluminescent probe $\left(\mathrm{pAb} \mathrm{b}_{2}-\mathrm{Ru}\right)$ at a concentration of $1 \mathrm{mg} \cdot \mathrm{mL}^{-1}$. The ECL detection was carried out in an open cell (see Experimental Section and Supporting Information), containing $100 \mathrm{mM}$ tripropylamine (TPA) in PBS. The mechanism of $\mathrm{ECL}$ generation with the sacrificial 
co-reactant TPA is already well known. ${ }^{4}$ Briefly, the TPA cationic radical ion, formed from direct oxidation of the co-reactant at the electrode, partly undergoes a deprotonation reaction, thus forming a highly reducing radical species, capable in turn of reducing $\mathrm{Ru}(\mathrm{bpy})_{3}{ }^{2+}$ to $\mathrm{Ru}-$ $(\mathrm{bpy})_{3}{ }^{+}$. The latter species then reacts with the oxidized co-reactant (that is continuously forming at the electrode), and in this way, through a simple potential step, the excited state, $\mathrm{Ru}(\mathrm{bpy})_{3}{ }^{2+*}$, is generated (Figure 3). The intensity of the ECL response is proportional to the concentration of the $\mathrm{Ru}(\mathrm{bpy})_{3}{ }^{2+}$ probe and, consequently, to the amount of biotin-palytoxin complexed to the sensor surface. Figure 4 shows the amperometric and ECL signals measured during a typical experiment with this immunosensor. ECL was obtained by pulsing the working electrode potential between 0 ( 1 s) and $1.45 \mathrm{~V}(0.5 \mathrm{~s})$ and recording simultaneously the current at the ITO substrate (black trace) and the ECL intensity (red trace) (Figure 4a). All experiments were repeated several times $(n \geq 10)$ in order to verify the stability of the measured ECL signal.

The current measured upon the application of the oxidation potential, following a typical Cottrellian behavior, is associated with the TPA oxidation, which, in turn, excites the $\mathrm{Ru}(\mathrm{bpy})_{3}{ }^{2+}$ probe, according to the mechanism described above (Figure 3e). An alternative pathway to ECL generation through the parallel oxidation of TPA and $\mathrm{Ru}(\mathrm{bpy})_{3}{ }^{2+}$ itself is very unlikely because of the large distance of the latter from the electrode surface, as predicted by Marcus theory. Figure $4 \mathrm{~b}$ shows the result of the blank experiment where the immunosensing surface was incubated with the labeled polyclonal antibody, in the absence of the toxin; in this case, while the same Cottrellian current was measured at the substrate and associated with the TPA oxidation, no ECL signal was detected, thus indicating totally negligible nonspecific interactions between $\mathrm{pAb}_{2}-\mathrm{Ru}$ and the immunosensor.

Construction of the Calibration Curve. Using the same device, biotin-PITX concentrations ranging from 0.13 to $260 \mathrm{ng} \cdot \mathrm{mL}^{-1}$ were analyzed (see Supporting Information Figure S10). In all cases, the steady-state ECL intensity was averaged upon three subsequent potential steps, and the results are shown in Figure 5a, black dots.

Data were analyzed by assuming that the $\mathrm{ECL}$ intensity was proportional to the biotin-PITX concentration through a typical adsorption isotherm governing the antibody-antigen recognition equilibrium:

$$
\mathrm{mAb}+n \cdot \mathrm{A} \underset{k_{\mathrm{d}}}{\stackrel{k_{\mathrm{a}}}{\rightleftharpoons}} \mathrm{mAb} \cdot \mathrm{A}_{\mathrm{n}}
$$

where A stands for antigen.

To fit the data, eq 1 was used: ${ }^{26}$

$$
l_{\mathrm{ECL}}=A \frac{B C_{\mathrm{PITX}}}{1+B C_{\mathrm{PITX}}}
$$

where $A$ is proportional to the maximum surface coverage and, therefore, to the maximum number of

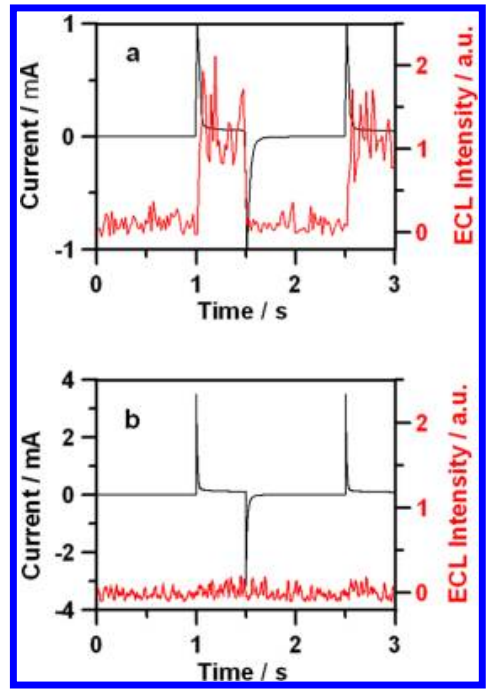

Figure 4. ECL intensity (red) and current (black) vs time, from PBS solution, $100 \mathrm{mM}$ TPA. Step potential $E_{1}=0 \mathrm{~V}, t_{1}=$ $1 \mathrm{~s} ; E_{2}=1.45 \mathrm{~V}, t_{2}=0.5 \mathrm{~s}$. PMT bias $750 \mathrm{~V}$, amplification 0.0 $\mathrm{nA}$. The MWCNT $-\mathrm{mA} \mathrm{b}_{1}$ were incubated with $260 \mathrm{ng} \cdot \mathrm{mL}^{-1}$ of biotin-PITX for $1 \mathrm{~h}$ (a) and without biotin-PITX (b) and treated with $2 \%$ BSA for $1 \mathrm{~h}$, followed by addition of $\mathrm{pAb}_{2}-$ Ru for $1 \mathrm{~h}$. Every step was followed by washing three times with $0.05 \%$ Tween-20 and PBS.

active antigen molecules adsorbed on the surface $\left(\Gamma_{\text {max }}\right)$, while $B$ represents the affinity constant between the antibody and its antigen. From the best fit (black trace), a value of $1 \times 10^{10} \mathrm{M}^{-1}$ was obtained for the affinity constant, in very good agreement with the corresponding value obtained from the SPR experiments with the free antibody (not bound to nanotubes), again confirming that the binding of the antibody to the MWCNTs does not induce significant changes to its specificity.

In order to highlight any specific role played by CNTs in the immunosensor performance, we prepared a second type of surface where the electrode modified with the electrografted polymer was directly incubated with the monoclonal antibody, i.e., lacking the CNT interlayer (Figure 1b). For the preparation of this control immunosensor, the same concentrations of $m A b_{1}$ and the same experimental conditions of the MWCNT- $m A b_{1}$-based immunoassay were used. If detected, variations in the performance of the control device should be ascribed to the specific role played by the CNT interlayer. Figure 5a shows that, with the conventional immunosensor, almost no ECL signal distinguishable from the background was detected at any concentration (red dots) when the same operating potential used above was applied (1.45 V). By contrast, a detectable signal was obtained, although significantly lower than in the case of MWCNT $-m A b_{1}$, when a higher potential, $\geq 2.6 \mathrm{~V}$, was applied at the interface (red dots). Such a behavior is in line with the known insulating properties of the poly(meth)acrylate films and would at the same time highlight the effective role played by the CNT interlayer in mediating the electron 


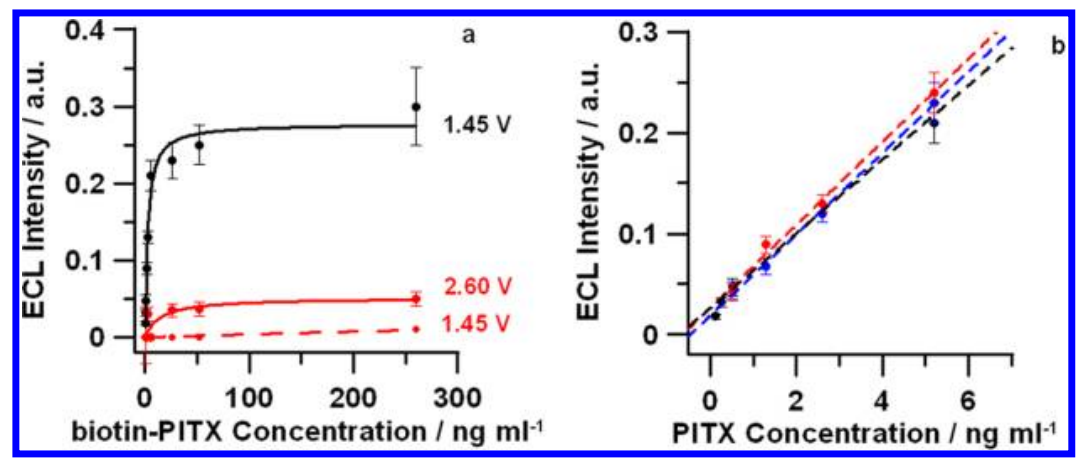

Figure 5. (a) ECL intensity for different concentrations of biotin-PITX $\left(260,52,26,5.2,2.6,1.3,0.52,0.26,0.13 \mathrm{ng} \cdot \mathrm{mL}^{-1}\right)$ in the presence of TPA at $100 \mathrm{mM}$ in PBS (pH 7.4). MWCNT-mAb -based immunoassay (black trace) at $1.45 \mathrm{~V}$. Conventional immunoassay (red trace) at $1.45 \mathrm{~V}$ (dashed line) and $2.60 \mathrm{~V}$ (solid line). (Potentials are reported $v s \mathrm{Ag} / \mathrm{Ag}^{+}$.) (b) ECL intensity for different concentrations of commercial PITX in linear dependence, co-reactant TPA $100 \mathrm{mM}$. PITX concentration in PBS: 0.13; $0.26 ; 0.52 ; 1.3 ; 2.6 ; 5.2 \mathrm{ng} \cdot \mathrm{mL}^{-1}$ (black dots). PITX in matrices $1: 10$ concentration: $0.52 ; 1.3 ; 2.6 ; 5.2 \mathrm{ng} \mathrm{mL}^{-1}$ (red dots for mussels and blue dots for microalgae). Electrochemiluminescence experimental conditions: PMT bias $750 \mathrm{~V}$, amplification $0.0 \mathrm{nA}$. Error bar shows the standard deviation $(n=3)$.

transfer between the conducting OTE substrate and the species in solution. In view of their well-known electrical and redox properties, ${ }^{27}$ the CNTs can in fact behave as individual nanoelectrodes, statistically interconnected and electrically wired to the ITO substrate, onto which the TPA oxidation process (as well as the direct fluorophore oxidation) may take place with great efficiency.

The fitting of the data points relative to the conventional immunosensor by eq 1 similarly provided both the surface coverage and affinity constant for such an interface. While almost the same value was obtained for the latter $\left(0.8 \times 10^{10} \mathrm{M}^{-1}\right)$, the amount of immobilized $\mathrm{mAb}_{1}$ was about 10 times lower than in the case of the MWCNT sensor, thus highlighting the fundamental role played by the nanotubes in the immobilization of large amounts of antibody in its active form.

In a second step, commercial PITX concentrations $\left(5.2,2.6,1.3,0.52,0.26,0.13 \mathrm{ng} \cdot \mathrm{mL}^{-1}\right)$ were analyzed, obtaining the same ECL intensity measured analyzing biotin-PITX (Figure 5b). Furthermore, the limits of detection (LOD) and quantification (LOQ) were obtained by the mean of ECL intensity of the PITX-free control plus 3 times and 10 times standard deviation, respectively. Using the fitting equation, we obtained for the nanostructured biosensor a LOD of $0.07 \mathrm{ng} \cdot \mathrm{mL}^{-1}$ and a LOQ of $0.24 \mathrm{ng} \cdot \mathrm{mL}^{-1}$. The LOD and LOQ obtained from the ECL platform were compared with those obtained with the most used analytical technique for the detection of PITX, liquid chromatography associated with mass spectrometry (see Supporting Information Figure S11). This comparison clearly shows that the LOD for the immunoassay-based biosensor is more than 300 times lower than that of the common approach used for the detection of the toxin. ${ }^{18}$

Although in the present work we have not tested the cross-reactivity with other PITX analogues, we have recently demonstrated the mAb1 specificity toward palytoxin, using a newly developed ELISA assay and the surface plasmon resonance technique. ${ }^{21}$ In particular, SPR data support a good affinity for palytoxins and their analogues ostreocin-D and 42-OH-palytoxin, with the following affinity order: palytoxin > ostreocin$\mathrm{D}>42-\mathrm{OH}$-palytoxin. ${ }^{21}$ Additionally, the ELISA assay did not allow the detection of various marine toxins (i.e., okadaic acid, yessotoxin, domoic acid, saxitoxin, and brevetoxin), which can be present in seafood together with PITX, at concentrations 4 orders of magnitude higher than that of the working range for PITX. ${ }^{21}$

Detection of PITX in Mussels and Microalgae Extracts. The antibody-CNT-based immunosensor was evaluated for its ability to detect PITX in shellfish and microalgae measuring the ECL signal in the relevant extracts spiked with different toxin concentrations.

Mussels and microalgae were extracted as previously described (see Experimental Section). ${ }^{28}$ In particular, mussels or microalgae extracts diluted in PBS (1:1, 1:10, and 1:100) were added with PITX at final concentrations of $0.52,1.30,2.60$, and $5.2 \mathrm{ng} \cdot \mathrm{mL}^{-1}$ and analyzed by the immunosensor in comparison to the same toxin concentrations in PBS without extract. The minimum dilution that did not interfere with the immunosensor was 1:10 for both matrices. Figure $5 b$ reports the $E C L$ signal, in the region of the linear dependence, measured analyzing PITX solutions in the presence of mussel or microalgae extracts (1:10), in comparison to that measured analyzing the same toxin concentrations without extracts. Interestingly, no difference has been observed between the ECL intensity and the trends for different matrices, within the standard deviation.

Furthermore, the LOD and LOQ for mussels were calculated as 0.05 and $0.22 \mathrm{ng} \cdot \mathrm{mL}^{-1}$, respectively, while the LOD and LOQ for microalgae were 0.06 and 0.23 $\mathrm{ng} \cdot \mathrm{mL}^{-1}$, respectively. These values, consistent with those found for PITX in PBS without extracts, indicate no matrix effect under these experimental conditions. 
This low matrix interference is the result of the synergistic combination of the CNT surface modification, which minimizes the unspecific adsorption, and the ECL transduction, intrinsic in the excited-state generation.

\section{CONCLUSIONS}

As supported by the results, we developed a highly sensitive ECL biosensor for the detection of palytoxin, obtaining very reproducible and reliable responses at each analyte concentration tested and reaching the LOD of $0.07 \mathrm{ng} \cdot \mathrm{mL}^{-1}$, with a LOQ of PITX in mussels $\left(\mathrm{LOQ}=0.22 \mathrm{ng} \cdot \mathrm{mL}^{-1}\right.$, corresponding to $2.2 \mu \mathrm{g} / \mathrm{kg}$ meat) that is more than 100 times lower in comparison to the LOQ $(228 \mu \mathrm{g} / \mathrm{kg})$ of the method currently used (LC-MS/MS), by using the same extraction procedure. ${ }^{27}$

The use of carbon nanotubes has dramatically contributed to the enhancement of the signal intensity produced by the device, in agreement with their renowned conductive and loading properties. Furthermore, the use of the immunosensor can dramatically reduce the time of analysis, very important for mussels, which have a very short shelf-life.

The high sensitivity of this device also in algal samples $\left(\mathrm{LOQ}=0.22 \mathrm{ng} \cdot \mathrm{mL}^{-1}\right)$ can be useful in monitoring programs to forecast shellfish contamination in the presence of potentially PITX-producing dinoflagellates, since Ostreopsis spp. do not always produce the same amounts of toxins, if any. The detection of PITX in net algal samples could give an earlier warning, reducing sanitary problems for the consumers as well as unnecessary economic losses for the producers. Further advantages of the construct, besides high sensitivity and stability over time, are the easiness of use, reusability, and the portability, thanks to its small size.

\section{EXPERIMENTAL SECTION}

Toxins and Other Materials. PITX (purity $>90 \%$ ) was purchased from Wako Chemicals GmbH (Neuss, Germany). The origin of other materials and chemicals is specified in the Supporting Information.

Preparation of the Immunogenic PITX Conjugate. PITX Activation. 4-(N-Maleimidomethyl)cyclohexanecarboxylic acid $N$-hydroxysuccinimide ester $(0.13 \mathrm{mg} ; 380 \mathrm{nmol})$ was dissolved in $0.1 \mathrm{~mL}$ of $\mathrm{N}, \mathrm{N}$-dimethylformamide, and the solution was added to PITX (0.2 mg; $80 \mathrm{nmol})$ dissolved in $1.7 \mathrm{~mL}$ of $0.1 \mathrm{M}$ phosphate buffer, $\mathrm{pH}$ 7.5). The solution was stirred for $8 \mathrm{~h}$ at room temperature. The resulting solution of activated PITX was directly used for the next step without further purification.

Carrier Protein Activation. Bovine serum albumin (2 mg) was dissolved in $0.8 \mathrm{~mL}$ of $25 \mathrm{mM}$ borate buffer $\mathrm{pH} 9.0$, and $0.21 \mathrm{mg}$ of 2-iminothiolane hydrochloride was added. The solution was stirred for $1 \mathrm{~h}$ at room temperature. The modified protein (BSA$\mathrm{SH})$ was applied to a G-25 column and eluted with $0.1 \mathrm{M}$ phosphate buffer $\mathrm{pH} 6.6$, containing $1 \mathrm{mM}$ EDTA, collecting 16-20 drop fractions and evaluating the protein concentration spectrophotometrically $(280 \mathrm{~nm})$.

Conjugation. Activated PITX was added in 50-fold molar excess to the activated carrier protein, and the solution was stirred for $1 \mathrm{~h}$ at room temperature and then dialyzed against $500 \mathrm{~mL}$ of PBS (140 mM NaCl, $2.7 \mathrm{mM} \mathrm{KCl}, 10 \mathrm{mM} \mathrm{Na}{ }_{2} \mathrm{HPO}_{4}$, $1.76 \mathrm{mM} \mathrm{KH}_{2} \mathrm{PO}_{4} ; \mathrm{pH} 7.3$ ) for 3 days, with regular changes of buffer every $4 \mathrm{~h}$. UV analysis $(\lambda B S A=248.8 \mathrm{~nm} ; \lambda \mathrm{PITX}=256.0 \mathrm{~nm})$ gave $9.23 \times 10^{-8} \mathrm{M}$ and $3.16 \times 10^{-6} \mathrm{M}$ BSA and PITX apparent concentrations, respectively. Thus, the molar ratio of PITX/BSA was estimated to be 34 .

Monoclonal Anti-PLTX Antibody Production. Mouse monoclonal anti-PLTX antibody 73D3 $\left(\mathrm{mAb}_{1}\right)$ was produced and purified from a hybridoma cell culture as previously described ${ }^{29}$ at the U.S. Army Medical Research Institute of Infectious Diseases (Fort Detrick, MD, USA).

Polyclonal Anti-PLTX Antibodies Production. Polyclonal anti-PITX antibodies were purified from the serum of rabbits immunized with PITX-BSA. Rabbit immunization was carried out at the University of Trieste, in conformity with Italian D.L. no. 116 of 27 January 1992 and associated guidelines in the European Communities Council Directive of 24 November 1986 (86/609 ECC) concerning animal welfare, and appendix A of the European Convention ETS 123. Male New Zealand white rabbits $(2 \mathrm{~kg}$; Harlan Laboratories; S. Pietro al Natisone, Italy) were kept in individual cages and acclimatized for one week before treatment. Animal room controls were set to maintain temperature $(21 \pm 1$ $\left.{ }^{\circ} \mathrm{C}\right)$ and humidity (60-70\%). Animals were fed with a standard commercial rabbit chow ad libitum (Harlan 2018, 3.4 kcal/g; Harlan Laboratories; S. Pietro al Natisone, Italy).

Rabbit polyclonal anti-PITX antibodies $\left(\mathrm{pAb}_{2}\right)$ were produced according to ref 29. Briefly, rabbits were immunized by two intradermal injections of $200 \mu \mathrm{g}$ of PITX-BSA (corresponding to about $116 \mu \mathrm{g}$ of PITX) diluted in PBS and emulsified in complete Freund's adjuvant $(1: 1 \mathrm{v} / \mathrm{v})$, within a month of each other. Rabbits were then boosted every month by both subcutaneous and intramuscular injections of 75 and $25 \mu \mathrm{g}$ of PITX-BSA, respectively, diluted in PBS and incomplete Freund's adjuvant (1:1 v/v). Test bleeds were taken 10 days after each booster injection. Rabbits were finally anaesthetized by intramuscular injection of Zoletil and xylazine (15 and $5 \mathrm{mg} / \mathrm{kg}$, respectively; Virbac S.r.l.; Milan, Italy) and exsanguinated, in accordance with national and international guidelines.

Polyclonal anti-PITX antibodies were affinity purified from rabbit sera diluted 1:10 (v/v) in $10 \mathrm{mM}$ Tris buffer $\mathrm{pH} 7.5$, using a $2 \mathrm{~mL}$ Sepharose $\mathrm{CNBr}(0.45 \mathrm{~g})$ immunoaffinity column. The gel was swelled in 15 volumes of $1 \mathrm{mM} \mathrm{HCl}$ at $0{ }^{\circ} \mathrm{C}$ and coupled with $3 \mathrm{mg}$ of PITX in $0.5 \mathrm{M}$ phosphate buffer $\mathrm{pH} 7.5$, overnight. After washing with two volumes of the same buffer, another washing step was carried out with $5 \mathrm{mM}$ phosphate buffer and $1 \mathrm{M} \mathrm{NaCl}$, $\mathrm{pH}$ 7.5. The gel was then saturated overnight with $100 \mathrm{mM}$ ethanolamine buffer $\mathrm{pH} 7.5$ at $0{ }^{\circ} \mathrm{C}$. Subsequent washing steps were carried out with 10 volumes of each of the following buffers: $10 \mathrm{mM}$ Tris $\mathrm{pH} 7.5,100 \mathrm{mM}$ glycine $\mathrm{pH}$ 2.5, $10 \mathrm{mM}$ Tris $\mathrm{pH} 8.8,100 \mathrm{mM}$ triethylamine $\mathrm{pH} 11.5$, and $10 \mathrm{mM}$ Tris $\mathrm{pH}$ 7.5.

Aliquots of $2 \mathrm{~mL}$ of rabbit immune serum were diluted 1:10 in $10 \mathrm{mM}$ Tris buffer $\mathrm{pH} 7.5$ and loaded on the Sepharose gel column for separation by gravity. Then, the column was washed with 20 volumes of $10 \mathrm{mM}$ Tris buffer $\mathrm{pH} 7.5$ and 20 volumes of $10 \mathrm{mM}$ Tris buffer pH 7.5 and $500 \mathrm{mM} \mathrm{NaCl}$. Glycine buffer (100 mM, pH 2.5) was used to elute the antibodies, collecting $1 \mathrm{~mL}$ fractions over $1 \mathrm{M}$ Tris buffer, $\mathrm{pH} 8.0$ (100 $\mu \mathrm{L}$ for each fraction), and the collected fractions were then dialyzed in $2 \mathrm{~L}$ of PBS overnight (6-8000 Da molecular weight cellulose membrane). Dialyzed fractions were concentrated using Amicon Ultra-15 centrifugal filter devices, following the manufacturer's directions, and final antibody concentration was evaluated in each fraction spectrophotometrically at $280 \mathrm{~nm}$.

Preparation of MWCNT $-m A b_{1}$ and $p A b_{2}-R u$. The synthesis of these conjugates is detailed in the Supporting Information. ${ }^{17}$

Electrografting of PNSA on ITO Surface. The electrochemically induced grafting was carried out in a single-compartment cell described in the Supporting Information by using ITO as a working electrode, platinum as counter electrode, and a silver spiral as a quasi-reference electrode. Electrografting was performed utilizing a solution of NSA $0.1 \mathrm{M}$ and TBAH $0.05 \mathrm{M}$ in $3 \mathrm{~mL}$ 
of DMF, scan rate $0.1 \mathrm{~V} \cdot \mathrm{s}^{-1}$. The potential was cycled until the reduction of the monomer $(-2.1 \mathrm{~V})$, after which the cycles were repeated. Before the polymerization the solution was degassed with argon for $15 \mathrm{~min}$, and then the measurement was carried out under argon flux.

Assembly of the Biosensor. A solution of MWNT-mAb $120 \mu \mathrm{L}$, $1 \mathrm{mg} \mathrm{mL}^{-1}$ ) containing $0.20 \mathrm{mg} \cdot \mathrm{mL}^{-1}$ of $\mathrm{mAb}$, together with a catalytic amount of EDCl, was dropped on the polymerized electrode and left on the surface overnight at $4{ }^{\circ} \mathrm{C}$. The concentration of the mAb ${ }_{1}$ coupled to MWCNTs was estimated by TGA (see Supporting Information). After incubation of MWCNT $-m A b_{1}$ the sandwich was assembled directly in the homemade electrochemical cell, which was used as such in an ELISA well. The optimized procedure consisted in the following series of operations: (i) the modified surface was covered with $40 \mu \mathrm{L}$ of a solution of BSA ( $2 \%$ in PBS) for $30 \mathrm{~min}$ at room temperature and abundantly washed with a solution of Tween-20 ( $0.05 \%$ in PBS); (ii) the substrate was incubated at $25^{\circ} \mathrm{C}$ for 60 min with $20 \mu \mathrm{L}$ of biotinylated PITX or PITX at the desired concentration and rinsed again with PBS; and (iii) the sensor was incubated with $20 \mu \mathrm{L}$ of the secondary antibody $\mathrm{pAb}_{2}-\mathrm{Ru}\left(1 \mathrm{mg} \mathrm{mL}^{-1}\right)$ for $60 \mathrm{~min}$ at room temperature. Unbound material was removed by washing repeatedly with $2 \%$ Tween- 20 $(\times 3)$ and PBS $(\times 3)$. The as-modified platform was characterized by scanning electron microscopy (see Supporting Information). For the conventional immunosensor setup, $20 \mu \mathrm{L}$ of a solution of $\mathrm{mAb}_{1}$ $\left(0.20 \mathrm{mg} \cdot \mathrm{mL}^{-1}\right)$, together with a catalytic amount of EDCl, was dropped on the polymerized electrode and left on the surface overnight at $4{ }^{\circ} \mathrm{C}$.

Electrochemiluminescence Detection. The ECL measurements were carried out in a PBS solution using $100 \mathrm{mM}$ TPA as a sacrificial co-reactant. The working electrode consisted of the modified ITO (as previously described) with a constant active area of $2 \mathrm{~cm}$ in diameter, while the counter electrode was a platinum spiral and the reference electrode was a homemade $\mathrm{Ag} / \mathrm{AgCl}$. The ECL signal generated by performing the potential step program was measured with a photomultiplier tube (PMT, Hamamatsu R4220p) placed, at a constant distance, under the cell and inside a dark box. A voltage in the range $550-750 \mathrm{~V}$ was supplied to the PMT. The light/current/voltage curves were recorded by collecting the preamplified PMT output signal (by a ultralow-noise Acton research model 181) with the second input channel of the ADC module of the AUTOLAB instrument. All the data reported in the calibration curve are the average of three independent experiments, while the error bars are the standard deviation (for $n=3$ within 10\%).

Detection of PITX in Mussel and Microalgae Extracts. To evaluate the applicability of the CNT-based immunosensor to detect PITX in shellfish and microalgae, samples of mussels and microalgae were collected and extracted as described below.

Samples. Mussels (Mytilus galloprovincialis) and microalgae were collected in the Gulf of Trieste (Italy). Mussel sample was obtained by homogenizing the shellfish meat $(200 \mathrm{~g})$ with an Ultra-Turrax (Ika-Werk; Staufen, Germany). Microalgae were collected by several vertical net hauls ( $20 \mu \mathrm{m}$ mesh) of seawater $\left(8 \mathrm{~L} ; 9.1 \times 10^{6}\right.$ algal cells $\left.\mathrm{L}^{-1}\right)$ free of Ostreopsis spp., containing mainly diatoms (Proboscia alata, Guinardia flaccida, Chaetoceros spp., and Hemiaulus hauckii, among the most abundant) and dinoflagellates (Ceratium fusus, C. furca, Dinophysis sacculus, Hermesinum adriaticum, and Protoperidinium spp., among the most abundant). The sample was centrifuged at $5000 \mathrm{rpm}$ for $20 \mathrm{~min}$, and the pellet was used as a microalgae sample for the analyses by the immunosensor. Mussels and microalgae samples were analyzed by high-resolution LC-MS/MS to verify the lack of PITX ${ }^{18,27}$ before their use in spiking experiments and toxin detection by the immunosensor.

Extract Preparation. Mussels $(1 \mathrm{~g})$ were extracted three times by homogenization with an Ultra-Turrax (14000 rpm, 3 min) with $3 \mathrm{~mL}$ of $80 \%$ aqueous $\mathrm{MeOH}$. After centrifugation at $5500 \mathrm{rpm}$ for $30 \mathrm{~min}$, the supernatants were pooled and the volume was adjusted to $10 \mathrm{~mL}$ with $80 \%$ aqueous $\mathrm{MeOH}$, obtaining $0.1 \mathrm{~g}$ of meat or microalgae equivalents per milliliter. ${ }^{27}$

Microalgae $(1 \mathrm{~g})$ were sonicated with $4 \mathrm{~mL}$ of $50 \%$ aqueous $\mathrm{MeOH}$ for $3 \mathrm{~min}$ in pulse mode, while cooling in an ice bath. The mixture was centrifuged at $5500 \mathrm{rpm}$ for $30 \mathrm{~min}$, the supernatant was decanted, and the pellet was washed twice with $3 \mathrm{~mL}$ of $50 \%$ aqueous $\mathrm{MeOH}$. The extracts were combined, and the volume was adjusted to $10 \mathrm{~mL}$ with $50 \%$ aqueous $\mathrm{MeOH}$, obtaining $0.1 \mathrm{~g}$ of microalgae equivalents $\mathrm{mL}^{-1}$.

Evaluation of Matrix Effect. To evaluate the matrix effect on PITX detection in mussels and microalgae, spiking experiments postextraction were carried out. Dilutions (1:1, 1:10, and 1:100) of PITX-free extracts of mussels or microalgae were spiked with known amounts of PITX in order to prepare, for each matrix dilution, a series of samples spiked at PITX levels ranging from 0.52 to $5.2 \mathrm{ng} \cdot \mathrm{mL}^{-1}$ for the analysis by the immunosensor.

Conflict of Interest: The authors declare no competing financial interest.

Acknowledgment. This work was supported by Regione Friuli Venezia-Giulia, Direzione Risorse Rurali, Agroalimentari e Forestali (Progetto "Kit e biosensori di elevata sensibilità per la determinazione delle tossine di alghe nelle acque e nei prodotti ittici del Friuli Venezia Giulia - Senstox"), and the European Union FP7 ANTICARB (HEALTH-2007-201587) program. Support is also acknowledged by the CNRS and the University of Trieste, the Italian Ministry of Education MIUR (Cofin Prot. 20085M27SS and Firb RBIN04HC3S). TEM images were recorded at the RIO Microscopy Facility Platform of Esplanade Campus (Strasbourg, France) and at the Electron Microscopy Facility of the University of Trieste (with the assistance of Mr. Claudio Gamboz). The authors wish to thank also Prof. G. Honsell (University of Udine) and Dr. M. Pelin (University of Trieste) for their assistance and discussions.

Supporting Information Available: Experimental procedures, detailing full characterization of the new compounds and the preparation and setup of the biosensor. This material is available free of charge via the Internet at http://pubs.acs.org.

Note Added after ASAP Publication: After this paper was published online August 30, 2012, corrections were made to Figure 3. The corrected version was reposted September 6, 2012.

\section{REFERENCES AND NOTES}

1. Moore, R. E.; Scheuer, J. P. Palytoxin: a New Marine Toxin from a Coelenterate. Science 1971, 172, 495-498.

2. Tubaro, A.; Durando, P.; Del Favero, G.; Ansaldi, F.; Icardi, G.; Deeds, J. R.; Sosa, S. Case Definitions for Human Poisonings Postulated to Palytoxins Exposure. Toxicon 2011, 57, 478495.

3. Riobó, P.; Franco, J. M. Palytoxins: Biological and Chemical Determination. Toxicon 2011, 57, 368-375.

4. Bard, A. J. Electrogenerated Chemiluminescence; Marcel Dekker: New York, 2004.

5. Richter, M. M. Electrochemiluminescence (ECL). Chem. Rev. 2004, 104, 3003-3036.

6. Forster, R. J.; Bertoncello, P.; Keyes, T. E. Electrogenerated Chemiluminescence. Annu. Rev. Anal. Chem. 2009, 2, 359385.

7. Hu, L. Z.; Xu, G. B. Applications and Trends in Electrochemiluminescence. Chem. Soc. Rev. 2010, 39, 3275-3304.

8. Katz, E.; Willner, I. Biomolecule-Functionalized Carbon Nanotubes: Applications in Nanobioelectronics. ChemPhysChem 2004, 5, 1085-1104.

9. Yang, W.; Ratinac, K. R.; Ringer, S. P.; Thordarson, P.; Gooding, J. J.; Braet, F. Carbon Nanomaterials in Biosensors: Should You Use Nanotubes or Graphene? Angew. Chem., Int. Ed. 2010, 49, 2114-2138.

10. Sardesai, N.; Pan, S.; Rusling, J. F. Electrochemiluminescent Immunosensor for Detection of Protein Cancer Biomarkers Using Carbon Nanotube Forest and $\left[\mathrm{Ru}-(\mathrm{bpy})_{3}\right]^{2+}$ Doped Silica Nanoparticles. Chem. Commun. 2009, 4968-4970.

11. Sardesai, N.; Barron, J. C.; Rusling, J. F. Carbon Nanotube Microwell Array for Sensitive Electrochemilunescent Detection of Cancer Biomarker Proteins. Anal. Chem. 2011, 83, 6698-6703.

12. Tasis, D.; Tagmatarchis, N.; Bianco, A.; Prato, M. Chemistry of Carbon Nanotubes. Chem. Rev. 2006, 106, 1105-1136.

13. Melle-Franco, M.; Marcaccio, M.; Paolucci, D.; Paolucci, F.; Georgakilas, V.; Guldi, D. M.; Prato, M.; Zerbetto, F. Cyclic 
Voltammetry and Bulk Electronic Properties of Soluble Carbon Nanotubes. J. Am. Chem. Soc. 2004, 126, 1646-1647.

14. Paolucci, D.; Melle Franco, M.; lurlo, M.; Marcaccio, M.; Prato, M.; Zerbetto, F.; Pénicaud, A.; Paolucci, F. Singling Out the Electrochemistry of Individual Single-Walled Carbon Nanotubes in Solution. J. Am. Chem. Soc. 2008, 130, 7393-7399.

15. Heller, D. A.; Jin, H.; Martinez, B. M.; Patel, D.; Miller, B. M.; Yeung, T.-K.; Jena, P. V.; Höbartner, C.; Ha, T.; Silverman, S. K.; et al. Multimodal Optical Sensing and Analyte Specificity Using Single-Walled Carbon Nanotubes. Nat. Nanotechnol. 2009, 4, 114-120.

16. Singh, P.; Campidelli, S.; Giordani, S.; Bonifazi, D.; Bianco, A.; Prato, M. Organic Functionalisation and Characterisation of Single-Walled Carbon Nanotubes. Chem. Soc. Rev. 2009, 38, 2214-2230.

17. Venturelli, E.; Fabbro, C.; Chaloin, O.; Ménard-Moyon, C.; Smulski, C. R.; Da Ros, T.; Kostarelos, K.; Prato, M.; Bianco, A. Antibody Covalent Immobilization on Carbon Nanotubes and Assessment of Antigen Binding. Small 2011, 7, 21792187.

18. Ciminiello, P.; Dell'Aversano, C.; Dello lacovo, E.; Fattorusso, E.; Forino, M.; Tartaglione, L. LC-MS of Palytoxin and Its Analogues: State of the Art and Future Perspectives. Toxicon 2011, 57, 376-389.

19. Alfonso, A.; Fernández-Araujo, A.; Alfonso, C.; Caramés, B.; Tobio, A.; Louzao, M. C.; Vieytes, M. R.; Botana, L. M. Palytoxin Detection and Quantification Using the Fluorescence Polarization Technique. Anal. Biochem. 2012, 424, 64-70.

20. Yakes, B. J.; DeGrasse, S. L.; Poli, M.; Deeds, J. R. Antibody Characterization and Immunoassays for Palytoxin Using an SPR Biosensor. Anal. Bioanal. Chem. 2012, 400, 2865-2869.

21. Boscolo, S.; Pelin, M.; De Bortoli, M.; Fontanive, G.; Barreras, A. Berti, F.; Sosa, S.; Chaloin, O.; Bianco, A.; Yasumoto, T.; et al. Sandwich ELISA Assay for the Quantification of Palytoxin and its Analogs in Natural Samples. Anal. Chim. Acta, submitted.

22. Jérôme, C.; Gabriel, S.; Voccia, S.; Detrembleur, C.; Ignatova, M.; Gouttebaron, R.; Jérôme, R. Preparation of Reactive Surfaces by Electrografting. Chem. Commun. 2003, 25002501.

23. Rapino, S.; Valenti, G.; Marcu, R.; Giorgio, M.; Marcaccio, M.; Paolucci, F. Microdrawing and Highlighting a Reactive Surface. J. Mater. Chem. 2010, 20, 7272-7275.

24. Yu, X.; Munge, B.; Patel, V.; Jensen, G.; Bhirde, A.; Gong, J. D.; Kim, S. N.; Gillespie, J.; Gutkind, J. S.; Papadimitrakopoulos, F.; et al. Carbon Nanotube Amplification Strategies for Highly Sensitive Immunodetection of Cancer Biomarkers. J. Am. Chem. Soc. 2006, 128, 11199-205.

25. Inuzuka, T.; Uemura, D.; Arimoto, H. The Conformational Features of Palytoxin in Aqueous Solution. Tetrahedron 2008, 64, 7718-7723.

26. McQuarrie, D. A.; Simon, J. D. Physical Chemistry: A Molecular Approach; University Science Books: Mill Valley, CA, 1997.

27. Hauquier, F.; Pastorin, G.; Hapiot, P.; Prato, M.; Bianco, A.; Fabre, B. Carbon Nanotube-Functionalized Silicon Surfaces with Efficient Redox Communication. Chem. Commun. 2006, 4536-4538.

28. Ciminiello, P.; Dell'Aversano, C.; Dello lacovo, E.; Fattorusso, E.; Forino, M.; Tartaglione, L.; Rossi, R.; Soprano, V.; Capozzo, D.; Serpe, L. Palytoxin in Seafood by Liquid Chromatography Tandem Mass Spectrometry: Investigation of Extraction Efficiency and Matrix Effect. Anal. Bioanal. Chem. 2011, 401, 1043-1050.

29. Bignami, G. S.; Raybould, T. J.; Sachinvala, N. D.; Grothaus, P. G.; Simpson, S. B.; Lazo, C. B.; Byrnes, J. B.; Moore, R. E.; Vann, D. C. Monoclonal Antibody-Based Enzyme-Linked Immunoassay for the Measurement of Palytoxin in Biological Samples. Toxicon 1992, 30, 687-700.

30. Ciminiello, P.; Dell'Aversano, C.; Fattorusso, E.; Forino, M.; Magno, G. S.; Tartaglione, L.; Grillo, C.; Melchiorre, N. The Genoa 2005 Outbreak. Determination of Putative Palytoxin in Mediterranean Ostreopsis ovata by a New Liquid Chromatography Tandem Mass Spectrometry Method. Anal. Chem. 2006, 78, 6153-6159. 